\title{
GOL'DBERG ORDER AND GOL'DBERG TYPE OF ENTIRE FUNCTIONS REPRESENTED BY MULTIPLE DIRICHLET SERIES
}

\author{
Md. Feruj Alam \\ Department of Mathematics, Rajshahi University of \\ Engineering \& Technology, Rajshahi
}

Received 28.06.07

Accepted 30.08.08

\begin{abstract}
We consider the Hadamard product of the class of entire multiple Dirichlet series in several complex variables having the same sequence of exponents. Our object is to study the nature of Gol'dberg order and Gol'dberg type of these functions.
\end{abstract}

\section{Notations}

The n-tuples $\left(\sigma_{1}, \cdots, \sigma_{n}\right),\left(m_{1}, \cdots, m_{n}\right),\left(s_{1}, \cdots, s_{n}\right)$ etc. of $C^{n}$ or $R^{n}$ will be denoted by their corresponding unsuffixed symbol $\sigma, m, s$ etc. respectively. By $I^{n}$ we shall mean the Cartesian product of $n$ copies of $I$ where $I$ is the set of non-negative integers.

$$
\begin{aligned}
& \text { For } s, w \in C^{n} \text { and } \alpha \in C \text { where, } \\
& s=\left(s_{1}, \cdots, s_{n}\right) \quad w=\left(w_{1}, \cdots, w_{n}\right)
\end{aligned}
$$

we define

(i) $s+w=\left(s_{1}+w_{1}, \cdots, s_{n}+w_{n}\right)$

(ii) $\alpha s=\left(\alpha s_{1}, \cdots, \alpha s_{n}\right)$

(iii) $S . W=s_{1} w_{1}+\cdots+s_{n} w_{n}$

For $a \in R, \quad \mathrm{~s} \in \mathrm{C}^{\mathrm{n}}$

$$
\text { (iv) } s+a=\left(s_{1}+a, \cdots, s_{n}+a\right)
$$

The positive hyperoctant $R_{+}^{n}$ in $R^{n}$ will be denoted by

$$
R_{+}^{n}=\left\{x: x \in R^{n}, \quad x_{i} \geq 0, j=1, \cdots, n\right\}
$$


For $t \in R_{+}^{n}$, we set $\|t\|=t_{1}+\cdots+t_{n}$.

For $k \in R, \bar{k}$ will denote the real n-tuple $(k, \cdots, k)$. For an entire function $f$ with domain $C^{n}, f^{k}$ will denote the function $\frac{\partial^{\|k\|} f}{\partial_{s_{1}}^{k_{1}} \cdots \partial_{s_{n}}^{k_{n}}}$, where $\mathrm{k} \in I^{n}$ and $f^{(\overline{0})}=f$.

We denote the n-tuple $\left(\lambda_{1 m_{1}}, \cdots, \lambda_{n m_{n}}\right)$ by $\lambda_{n, m}$

Thus, $\quad s . \lambda_{n, m}=s_{1} \lambda_{1 m_{1}}+\cdots+s_{n} \lambda_{n m_{n}}$.

\section{Introduction}

We consider the multiple Dirichlet series

$$
\begin{aligned}
& f\left(s_{1}, \cdots, s_{n}\right)=\sum_{m_{1}, \cdots, m_{n}=1}^{\infty} a_{m_{1} \cdots m_{n}} \exp \left\{s_{1} \lambda_{1 m_{1}}+\cdots+s_{n} \lambda_{n m_{n}}\right\}, \text { that is, } \\
& f(s)=\sum_{m=1}^{\infty} a_{m} \exp \left\{s . \lambda_{n, m}\right\} . \\
& \left(s_{j}=\sigma_{j}+i \tau_{j} \in C, \quad j=1, \cdots, n\right), a_{m} \in C, \quad \text { and }\left\{\lambda_{j m_{j}}\right\}_{m_{j}=1}^{\infty}, j=1, \cdots, n \text { are } n
\end{aligned}
$$

sequences of exponents satisfying the conditions

$0<\lambda_{j 1}<\lambda_{j 2}<\cdots<\lambda_{j k} \rightarrow \infty$ as $k \rightarrow \infty, \quad$ for $j=1, \cdots, n$.

Throughout we assume that $\lim _{m_{j} \rightarrow \infty} \frac{\log m_{j}}{\lambda_{j} m_{j}}=0, \quad j=1, \cdots, n$

If (2.3) holds then the domain of convergence of the series (2.1) coincides with its domain of absolute convergence [1].

All the multiple Dirichlet series of the form (2.1) having the same sequence of exponents $\left\{\lambda_{j m_{j}}\right\}_{m_{i}=1}^{\infty}, j=1, \cdots, n$. satisfying (2.2) are absolutely and uniformly convergent in $C^{n}$ and hence are entire functions.

For the entire functions $f$ and $g$, we define Hadamard product [2] $f * g$ by

$$
f(s) * g(s)=\sum_{m=1}^{\infty} a_{m} b_{m} \exp \left\{s . \lambda_{n, m}\right\}
$$


where $f(s)=\sum_{m=1}^{\infty} a_{m} \exp \left\{s . \lambda_{n, m}\right\}$ and $g(s)=\sum_{m=1}^{\infty} b_{m} \exp \left\{s . \lambda_{n . m}\right\}$

For $k \in I^{n}$, we define

$$
\begin{aligned}
& f^{k}(s)=\sum_{m=1}^{\infty} \lambda_{n, m}^{k} a_{m} \exp \left\{s . \lambda_{n, m}\right\} \\
& f^{k}(s) * g^{k}(s)=\sum_{m=1}^{\infty} \lambda_{n, m}^{2 k} a_{m} b_{m} \exp \left\{s . \lambda_{n, m}\right\}
\end{aligned}
$$

Definitions : We define the poly half plane $D_{l}$ as $D_{l}=\left\{S: S \in C^{n}, \operatorname{Re} s=\sigma<<l\right\}$, where $l \in R^{n}$. These type of domains are called the fundamental domains. The region $D_{l}+r$, depending on the parameter $r \in R$, is defined as $D_{l}+r=\left\{s+r, s \in D_{l}\right\}$. We simply write $\mathrm{D}$ instead of $D_{l}$. Then for the entire function $f$, given by (2.1), we define the maximum modulus $M_{f, D}(r)$ with respect to the region $\mathrm{D}$, where $r \in R$ as

$$
M_{f, D}(r)=\sup \{|f(s)|: s \in D+r\} .
$$

Let $f$ be an entire function and D be a fundamental domain. Also, let $S_{f}$ be the set of points $\alpha \in R$ such that for every $\alpha \in S_{f}$, there corresponding an $r_{0} \in R$ such that

$$
\log M_{f, D}(r) \leq e^{r \alpha}, \text { for } r \geq r_{0} .
$$

The infimum of the set $S_{f}$ is called the Gol'dberg order $\rho(D)$ of $f$ with respect to the region D. We say that $f$ is of infinite or finite Gol'dberg order according as $S_{f}$ is empty or non-empty.

Next, for the Gol'dberg order $\rho(D)>0$, let $K_{f}(\rho)$ be the set of all $K \in R$ such that

$$
\log M_{f, D}(r) \leq K e^{r \alpha}, \text { for } r \geq r_{0} .
$$

The infimum of the set $K_{f}(\rho)$ is called the Gol'dberg type $T(D)$ of $f$ corresponding to $\rho(D)$. As before, we say that $f$ is of infinite or finite Gol'dberg type according as $K_{f}(\rho)$ is empty or non-empty. We shall call Gol'dberg order and Gol'dberg type simply as Gorder and G-type respectively.

From the definition it follows easily that 


$$
\begin{aligned}
& \rho(D)=\lim _{r \rightarrow \infty} \sup \frac{\log \log M_{f, D}(r)}{r} \\
& \rho_{k}(D)=\lim _{r \rightarrow \infty} \sup \frac{\log \log M_{f^{k}, D}(r)}{r} \\
& T(D)=\limsup _{r \rightarrow \infty} \frac{\log M_{f, D}(r)}{e^{r \rho(D)}}, \quad \text { if } \rho(D)>0 \\
& T_{k}(D)=\lim _{r \rightarrow \infty} \sup \frac{\log M_{f^{k}, D}(r)}{e^{r \rho_{k}(D)}}, \quad \text { if } \underset{k}{\rho(D)>0}
\end{aligned}
$$

We know that the G-order $\rho(D)$ does not dependent on the choice of the domain $D$ while G-type $T(D)$ does [3]. Here we may write $\rho$ instead of $\rho(D)$. It is also know [3] that

$$
\begin{aligned}
& \rho=\lim _{m \rightarrow \infty} \sup \frac{\left\|\lambda_{n, m}\right\| \log \left\|\lambda_{n, m}\right\|}{-\log \left|a_{m}\right|} \\
& \underset{k}{\rho}=\lim _{m \rightarrow \infty} \sup \frac{\left\|\lambda_{n, m}\right\| \log \left\|\lambda_{n, m}\right\|}{-\log \left|a_{m} \lambda_{n, m}^{k}\right|}
\end{aligned}
$$

Theorem 1: The function $f^{k}(s) * g^{k}(s)$, as defined by (2.6) is an entire function.

Proof : Since $f(s)$ and $g(s)$ are entire functions so $f^{k}(s)$ and $g^{k}(s)$ are also entire functions. Now $f^{k}(s)=\sum_{m=1}^{\infty} \lambda_{n, m}^{k} a_{m} \exp \left\{s . \lambda_{n, m}\right\} \quad$ [defined by (2.5)]. But the series $\sum_{m=1}^{\infty}\left|\lambda_{n, m}^{k} a_{m}\right| \exp \left\{\sigma . \lambda_{n, m}\right\}$ is convergent for all $\sigma \in R^{n}$. In particular, it is convergent at $\sigma=\overline{0}$, so that $\sum_{m=1}^{\infty}\left|\lambda_{n, m}^{k} a_{m}\right|$ is convergent. Thus $\lim _{m \rightarrow \infty}\left|\lambda_{n, m}^{k} a_{m}\right|=0$ and hence the nsequence $\left\{\left|\lambda_{n, m}^{k} a_{m}\right|\right\}$ is bounded. Also, the series

$$
\sum_{m=1}^{\infty}\left|\lambda_{n, m}^{k} b_{m}\right| \exp \left\{\sigma \cdot \lambda_{n, m}\right\}
$$

is convergent for all $\sigma \in R^{n}$ and consequently,

$$
\sum_{m=1}^{\infty} \lambda_{n, m}^{2 k} a_{m} b_{m} \exp \left\{\sigma \cdot \lambda_{n, m}\right\}
$$


is convergent for all $\sigma \in R^{n}$. This implies that

$$
\sum_{m=1}^{\infty} \lambda_{n, m}^{2 k} a_{m} b_{m} \exp \left\{s . \lambda_{n, m}\right\}
$$

is absolutely convergent for all $s \in C^{n}$

Hence $f^{k}(s) * g^{k}(s)$ defined by (2.6) represents an entire function.

Theorem 1.1.2: Let $f$ and $g$ be entire functions where $f^{k}(s)=\sum_{m=1}^{\infty} \lambda_{n, m}^{k} a_{m} \exp \left\{s . \lambda_{n, m}\right\}$ and $g^{k}(s)=\sum_{m=1}^{\infty} \lambda_{n, m}^{k} b_{m} \exp \left\{s . \lambda_{n, m}\right\}$ having G-order $\rho_{k_{f}}\left(0<\rho_{k_{f}}<\infty\right)$ and $\rho_{k_{g}}\left(0<\rho_{k_{g}}<\infty\right)$ respectively. Then $f^{k}(s) * g^{k}(s)$ is an entire function with G-order $\rho_{k}$ such that $\rho_{k} \leq\left(\rho_{k_{f}} \rho_{k_{g}}\right)^{1 / 2}$ provided $\log \frac{1}{\left|\lambda_{n, m}^{2 k} a_{m} b_{m}\right|} \sim\left\{\log \frac{1}{\left|\lambda_{n, m}^{k} a_{m}\right|} \log \frac{1}{\left|\lambda_{n, m}^{k} b_{m}\right|}\right\}^{1 / 2}$

Proof: $f^{k}(s) * g^{k}(s)$ is an entire function by theorem 1 . Now by (2.12)

$$
\begin{aligned}
& \frac{1}{\rho_{k_{f}}}=\lim _{m \rightarrow \infty} \inf \frac{-\log \left|\lambda_{n, m}^{k} a_{m}\right|}{\left\|\lambda_{n, m}\right\| \log \left\|\lambda_{n, m}\right\|} \text { and } \\
& \frac{1}{\rho_{k_{g}}}=\lim _{m \rightarrow \infty} \inf \frac{-\log \left|\lambda_{n, m}^{k} b_{m}\right|}{\left\|\lambda_{n, m}\right\| \log \left\|\lambda_{n, m}\right\|}
\end{aligned}
$$

For arbitrary $\varepsilon>0$, it follows that

$$
\begin{aligned}
& \frac{1}{\rho}-\varepsilon / 2<\frac{\log \frac{1}{\left|\lambda_{n, m}^{k} a_{m}\right|}}{\left\|\lambda_{n, m}\right\| \log \left\|\lambda_{n, m}\right\|} \text { and } \\
& \frac{1}{\rho}-\varepsilon / 2<\frac{\log \frac{1}{\| \lambda_{k_{s}}^{k} b_{m} \mid}}{\left\|\lambda_{n, m}\right\| \log \left\|\lambda_{n, m}\right\|}
\end{aligned}
$$

Now we can write

$$
\frac{\log \frac{1}{\left|\lambda_{n, m}^{k} a_{m}\right|} \log \frac{1}{\left|\lambda_{n, m}^{k} b_{m}\right|}}{\left(\left\|\lambda_{n, m}\right\| \log \left\|\lambda_{n, m}\right\|\right)^{2}}>\left(\frac{1}{\rho}-\varepsilon / 2\right)\left(\frac{1}{\rho_{,}}-\varepsilon / 2\right)
$$


or, $\quad \frac{\left\{\log \frac{1}{\mid \lambda_{n, m}^{k} a_{m}} \log \frac{1}{\left|\lambda_{n, m}^{k} b_{m}\right|}\right\}^{1 / 2}}{\left\|\lambda_{n, m}\right\| \log \left\|\lambda_{n, m}\right\|}\left\{\left(\frac{1}{\rho_{k_{f}}}-\varepsilon / 2\right)\left(\frac{1}{\rho_{k_{g}}}-\varepsilon / 2\right)\right\}^{1 / 2}$

for sufficiently large $\|\mathrm{m}\|$.

Now if $\quad \log \frac{1}{\left|\lambda_{n, m}^{2 k} a_{m} b_{m}\right|} \sim\left\{\log \frac{1}{\left|\lambda_{n, m}^{k} a_{m}\right|} \log \frac{1}{\left|\lambda_{n, m}^{k} b_{m}\right|}\right\}^{1 / 2}$

then $\quad \frac{\log \frac{1}{\left|\lambda_{n, m}^{2 k} a_{m} b_{m}\right|}}{\left\|\lambda_{n, m}\right\| \log \left\|\lambda_{n, m}\right\|}>\left\{\left(\frac{1}{\rho_{k_{f}}}-\varepsilon / 2\right)\left(\frac{1}{\rho_{k_{s}}}-\varepsilon / 2\right)\right\}^{\frac{1}{2}}$

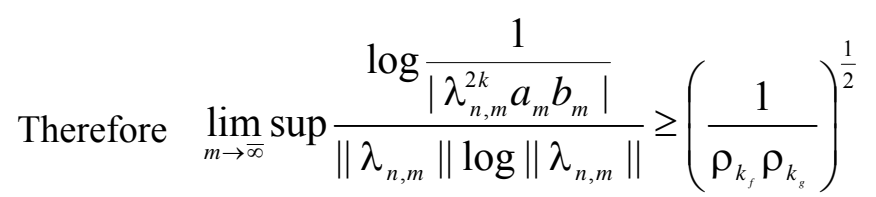

Thus $\quad \frac{1}{\rho} \geq\left(\frac{1}{\rho_{k_{f}} \rho_{k_{g}}}\right)^{\frac{1}{2}}$

Hence $\quad \rho_{k} \leq\left(\rho_{k_{f}} \rho_{k_{g}}\right)^{1 / 2}$.

Theorem 3: Let $f^{k}$ and $g^{k}$ be entire function of G-order $\rho_{k_{f}}\left(0<\rho_{k_{f}}<\infty\right)$ and $\rho_{k_{g}}\left(0<\rho_{k_{g}}<\infty\right)$ and finite G-type $T_{k_{f}}(D)$ and $T_{k_{g}}(D)$ respectively having the same fundamental domain D. If $f^{k} * g^{k}$ is of G-order $\rho_{k}\left(0<\rho_{k}<\infty\right)$, where $\log M_{f^{k} * g^{k}}(r) \sim \log M_{f^{k}, D}(r) \log M_{g^{k}, D}(r)$, then $\rho_{k} \leq \rho_{k_{f}}+\rho_{k_{g}}$.

Also if $T_{k}(D)$ be the G-type of $f^{k} * g^{k}$ with respect to the domain $\mathrm{D}$ then $T_{k}(D) \leq T_{k_{f}}(D) T_{k_{g}}(D)$ provided the sign of equality holds in $\rho_{k} \leq \rho_{k_{f}}+\rho_{k_{g}}$.

Proof: From (2.8), we have

$$
\rho_{k_{f}}(D)=\lim _{r \rightarrow \infty} \sup \frac{\log \log M_{f^{k}, D}(r)}{r} \text { and } \rho_{k_{g}}(D)=\lim _{r \rightarrow \infty} \sup \frac{\log \log M_{g^{k}, D}(r)}{r}
$$


Hence for an arbitrary $\varepsilon>0$,

$$
\begin{aligned}
& \log M_{f^{k}, D}(r)<\exp \left\{r\left(\rho_{k_{f}}+\varepsilon / 2\right)\right\}, \quad \text { for } r>r_{0} \text { and } \\
& \log M_{g^{k}, D}(r)<\exp \left\{r\left(\rho_{k_{g}}+\varepsilon / 2\right)\right\}, \text { for } r>r_{0} .
\end{aligned}
$$

Hence for $r>r_{0}$, we have

$$
\log M_{f^{k}, D}(r) \log M_{g^{k}, D}(r)<\exp \left\{r\left(\rho_{k_{f}}+\rho_{k_{g}}+\varepsilon\right)\right\}
$$

Thus if $\log M_{f * g, D}(r) \sim \log M_{f^{k}, D}(r) \log M_{g^{k}, D}(r)$, then

$$
\rho_{k}(D)=\lim _{r \rightarrow \infty} \sup \frac{\log \log M_{f^{*} * g^{k}, D}(r)}{r} \leq \rho_{k_{f}}+\rho_{k_{g}}
$$

That is, $\rho_{k} \leq \rho_{k_{f}}+\rho_{k_{g}}$.

Again from (2.10) we have

$$
\frac{\log M_{f^{k}, D}(r)}{e^{r \rho_{k_{f}}}}<T_{k_{f}}(D)+\varepsilon, \quad \text { for } r>r^{\prime} \text { and }
$$

$$
\frac{\log M_{g^{k}, D}(r)}{e^{r \rho_{k_{g}}}}<T_{k_{g}}(D)+\varepsilon, \quad \text { for } r>r^{\prime}
$$

Hence,

$$
\frac{\log M_{f^{k}, D}(r) \log M_{g^{k}, D}(r)}{e^{r\left(\rho_{k_{f}}+\rho_{k_{g}}\right)}}<\left[T_{k_{f}}(D)+\varepsilon\right]\left[T_{k_{g}}(D)+\varepsilon\right], \text { for } r>r^{\prime}
$$

Thus if, $\rho_{k}=\rho_{k_{f}}+\rho_{k_{g}}$

$$
\lim _{r \rightarrow \infty} \sup \frac{\log M_{f^{k_{*} k^{k}, D}}(r)}{e^{r_{\rho_{k}}}} \leq T_{k_{f}}(D)+T_{k_{g}}(D)
$$

That is $T_{k}(D) \leq T_{k_{f}}(D) T_{k_{g}}(D)$.

\section{Acknowledgement}

I express my sincere gratitude to my respected supervisor Professor Dr. Md. Muklesur Rahman, Department of Mathematics, University of Dhaka, for his valuable suggestions and guidance in the preparation of this paper. 


\section{REFERENCES}

1. Janusauskas, A.I. Elementary theorems on the convergence of double Dirichlet series. Soviet Math. Dokl. Vol. 18, No. 3 pp 610-614, (1977).

2. Titchmarsh. E. C. The Theory of Functions. Oxford univ. press, pp 157-158, (1932).

3. Sarker. P.K. On Gol'dberg order and gol'dberg type of an entire function of several complex variables represented by multiple Dirichlet series. Indian J. of pure and App. Math. 13 (10), pp 1221-1229, 1982. 